\title{
INVESTIGACIONES
}

\section{Intención y práctica con TIC en formadores de profesores: Congruencias, colisiones y autoeficacia*}

\author{
Intention and ICT practices in teacher trainers: \\ Congruencies, collisions and self-efficacy
}

\author{
José Miguel Garrido-Miranda \\ Pontificia Universidad Católica de Valparaíso \\ Correo electrónico: jgarrido@pucv.cl
}

\begin{abstract}
RESUMEN
Un desafío de la formación inicial de docentes es preparar para desempeñarse en una sociedad mediada por lenguajes, interacciones y ambientes digitalizados. Los estudios señalan, sin embargo, que los egresados de pedagogía no logran la autoeficacia para utilizar TIC pedagógicamente, entre otras razones, por las pocas oportunidades, ejemplos o modelamientos propiciados por sus formadores; ¿muestra esto la baja autoeficacia de los propios formadores? Para responder, este estudio analiza las intenciones y prácticas pedagógicas con TIC de 116 formadores de la carrera de educación básica, utilizando para ello un diseño mixto, secuencial y exploratorio con aplicación de un cuestionario estandarizado y entrevistas semiestructuradas. Los resultados muestran diferentes maneras de incorporar las TIC producto de las creencias sobre el protagonismo de profesores y estudiantes, y sobre el uso colaborativo o reproductivo de las tecnologías. Se discuten las congruencias y colisiones de estas creencias y cómo influyen en la autoeficacia de los docentes-formadores.
\end{abstract}

Palabras clave: autoeficacia, formador de formadores, actitudes de profesores, métodos de enseñanza, creencias pedagógicas, usos de tecnologías en educación

\begin{abstract}
A challenge of the initial formation of teachers is to prepare to perform in a society mediated by languages, interactions, and digitalized environments. The studies suggest, however, that pedagogy graduates do not acquire self-efficacy to use ICT pedagogically, among other reasons, for the few opportunities, examples or modeling provided by their teacher-trainers; Does this show the low self-efficacy of the trainers themselves? To answer, this study analyzes the intentions and pedagogical practices with ICT of 116 trainers of basic education, using a mixed, sequential and exploratory design with the application of a standardized questionnaire and semi-structured interviews. The results show different ways of incorporating ICT as a product of beliefs about the role of teachers and students, and about the collaborative or reproductive use of technologies. The congruences and collisions of these beliefs are discussed and how they influence the self-efficacy of teacher-trainers.
\end{abstract}

Key words: self-efficacy, teacher's trainer, teacher attitudes, teaching methods, pedagogical beliefs, technology uses in education 


\section{INTRODUCCIÓN}

Disponer de tecnologías de información y comunicación (TIC) en las aulas y alfabetizar tecnológicamente a los actores educativos, son insuficientes para propiciar prácticas innovadoras y efectivas de enseñanza para el aprendizaje (Suaréz, Almerich, Gargallo \& Aliaga, 2013). Esto porque las diferencias ecosistémicas de cada contexto formativo y el papel de las posiciones pedagógico-curriculares manifestadas por los docentes al momento de decidir qué y cómo utilizarlas en su desempeño profesional, originan diversas formas no predictibles de interacción entre las personas y las tecnologías (Aprea, 2006; Cuban, Kikpatrick \& Peck, 2001; Duart, Gil, Pujol \& Castaño, 2008; Sancho, 2012; van den Beemt \& Diepstraten, 2016; Zhao \& Frank, 2003).

Considerando el reto que significa formar profesores que coadyuven a las nuevas generaciones a desarrollar las capacidades para desenvolverse cultural, cognitiva y éticamente en una sociedad compleja, globalizada, competitiva, inequitativa y mediada por el uso de lenguajes, ambientes e interacciones de tipo digital (Den Exter, Rowe, Boyd \& Lloyd, 2012; Lim, Chai \& Churchill, 2010; Newell, Pembroke \& Boyd, 2012), es necesario preguntarse por el papel de la formación inicial de docentes (FID) en la preparación del nuevo profesorado para incorporar las tecnologías digitales en su desempeño profesional. Diversos informes e investigaciones advierten que la FID ofrece pocas experiencias para aprender a utilizar formativamente las tecnologías digitales a los profesores en formación, influyendo en los bajos niveles de autoeficacia que éstos manifiestan para utilizarlas pedagógicamente (Enochsson \& Rizza, 2009; Liaw, 2017; Tondeur, Pareja Roblin, van Braak, Voogt \& Prestridge, 2016).

En este marco, un aspecto necesario de profundizar es la intencionalidad pedagógica que los docentes-formadores de carreras de pedagogía dan a las TIC en sus prácticas de enseñanza, y cómo éstas dan cuenta de su propia autoeficacia para incorporarlas formativamente. Este es el propósito del presente estudio, el cual mediante un diseño de investigación mixto secuencial y exploratorio, analiza, caracteriza y compara las disposiciones pedagógicas y tipo de prácticas con TIC informadas por 116 docentesformadores de la carrera de educación básica en 21 universidades chilenas.

Los resultados y posterior discusión permiten observar que las decisiones de los docentes-formadores para incorporar TIC en sus prácticas son producto de las creencias sobre el protagonismo que debe asumir o el propio docente o los estudiantes en el desarrollo de los procesos de enseñanza y aprendizaje; y sobre el aporte colaborativo o reproductivo que esperan del uso de las tecnologías. Esto origina congruencias y colisiones en el sistema de creencias de los docentes-formadores que influyen sobre su propia autoeficacia para modelar pedagógicamente el uso de las TIC al nuevo profesorado.

\section{MARCO REFERENCIAL}

\subsection{FORMACIÓN INICIAL DE DOCENTES Y LAS TIC}

Diversos informes e investigaciones advierten que la FID ofrece a los profesores en formación, pocas experiencias para aprender a utilizar pedagógica y didácticamente las tecnologías digitales, provocando bajos niveles de autoeficacia entre aquellos para utilizarlas 
en actividades formativas (Enochsson \& Rizza, 2009; Lee \& Lee, 2014; Liaw, 2017; Tondeur et al., 2016). Si bien, hay buena disposición para usarlas, tanto los profesores en formación como los profesores principiantes, evidencian diferencias importantes en la intensidad y calidad de los usos de TIC vivenciados durante su formación inicial, al comparar los usos para tareas personales o de preparación de clases, y los usos para incorporarlas de manera efectiva en las interacciones y prácticas realizadas en el aula (Liaw, 2017; Valtonen et al., 2015; van den Beemt \& Diepstraten, 2016). Al respecto, los profesores en formación demuestran dificultades para transferir sus conocimientos tecnológicos a sus prácticas de aula (Hammond et al., 2009).

Considerando que la evidencia señala que quienes estudian una carrera de pedagogía son, comparativamente, los profesionales en formación que menor uso hacen de las TIC durante su etapa formativa (Enochsson \& Rizza, 2009) o bien, que sus experiencias de uso resultan poco innovadoras (Brun, 2011; Flores-Lueg \& Roig Vilab, 2016), adquiere relevancia el papel de los formadores de formadores para modelar, motivar y ofrecer oportunidades para aprender a usar las TIC de manera efectiva, crítica y reflexiva (Hammond et al., 2009; Tondeur et al., 2016; Tondeur et al., 2012; Valtonen et al., 2015), propiciando a través de ello, que los profesores en formación desarrollen las habilidades y destrezas necesarias (Tondeur et al., 2012).

Para esto, se requiere fortalecer el ámbito de conocimiento pedagógico de las TIC a lo largo de la formación inicial de docentes tanto en número como en calidad de las experiencias que se desarrollan. Para lo cual, tanto las asignaturas específicas destinadas al aprendizaje educativo de las tecnologías digitales, como otras asignaturas de los curriculum de formación inicial, deben incorporar explícitamente modelos pedagógico-didácticos para su uso en las aulas de clase (Sang, Tondeur, Chai \& Dong, 2016; Tondeur et al., 2012), propiciando con ello que los futuros profesores construyan protocolos de actuación profesional, en el sentido planteado por Schön (1992), para la incorporación de TIC con carácter contextual e innovador.

\subsection{CREENCIAS PEDAGÓGICAS Y TECNOLOGÍAS DIGITALES}

El estudio de las creencias, es parte del análisis a la relación entre pensamiento y acción que se vive en las aulas; asume el papel determinante del pensamiento subjetivo en el proceso de toma de decisión realizado por el profesorado respecto del eje curriculum-contexto-práctica (Clark \& Peterson, 1986; Cortez, Fuentes, Villablanca \& Guzmán, 2013; Valverde, Fernández \& Revuelta, 2013). A pesar del abundante número de investigaciones sobre el tema, no hay acuerdo sobre su influencia causal en la actuación práctica de los docentes (Kane, Sandretto \& Heath, 2002; Lim \& Chai, 2008). Quienes defienden la conexión causal y congruente entre pensamiento y la acción, plantean que la creencia no es un conocimiento aislado o simple sobre algo, sino que la resultante de la confluencia entre un contexto, un contenido y la persona, cuya consecuencia es la configuración de un "sistema de creencias" que actúa como un filtro, restringiendo o ampliando la percepción, el comportamiento y el juicio aplicado por el sujeto en sus prácticas habituales (Ertmer, Ottenbreit-Leftwich \& Tondeur, 2015; Kagan, 1992). Este sistema de creencias está conformado por las creencias-anclas (anchors believes) que favorecen o dificultan la práctica de aprendizaje, al actuar como un anclaje en la relación pensamiento-acción; y las creencias de naturaleza más frágil (brittle believes) que se vinculan con mayor o menor intensidad y continuidad con las creencias de anclaje (Kagan, 1992). 
En este marco, surgen las denominadas "creencias pedagógicas" o pensamientos que influyen y determinan las disposiciones e intenciones de los docentes para organizar e implementar la acción formativa. Se distinguen básicamente dos, una vinculada a un enfoque directivo que se manifiesta en prácticas de aula con un rol protagónico del docente y una actuación más bien pasiva del estudiante; mientras que la otra responde a un enfoque constructivista manifestada en prácticas de aula con un papel protagónico de los estudiantes y un rol mediador del docente (Ertmer et al., 2015; Lim \& Chai, 2008). A esta clasificación, otros autores han agregado dos enfoques adicionales, el de aprendizaje activo y el de aprendizaje social (Lin, Wang \& Lin, 2012).

En cuanto al pensamiento y acción pedagógica con TIC, las investigaciones concluyen que las creencias pedagógicas del profesorado resultan más decidoras sobre aquella decisión, que el potencial y oportunidad de innovación que la propia tecnología ofrece (Arancibia, Soto \& Contreras, 2010; Jimoyiannis \& Komis, 2007; Lim \& Chai, 2008; Rienties, Brouwer \& Lygo-Baker, 2013). Esto implica que la evaluación final que realiza el docente sobre el aporte o dificultad pedagógica que una herramienta digital tiene para su contexto y/o materia de clases, influye más que su percepción sobre el potencial que aquella ofrece desde la dimensión tecnológica, originando así disposiciones de negación, resistencias o disposición para incorporarlas (Arancibia et al., 2010; Gargallo, Suárez \& Almerich, 2006; Sadaf, Newby \& Ertmer, 2012). Por otro lado, la modificación de las creencias pedagógicas está vinculada directamente a la participación en experiencias vivenciales o nuevas prácticas donde el profesorado aprehende formas de actuación enriquecidas y complejas cognitivamente, en las cuales observa e integra aspectos del curriculum, la evaluación, los contenidos disciplinarios, la didáctica y el uso de medios o recursos, a partir de las cuales se asientan como parte de su pensamiento, operando como base de la disposición (creencia ancla) para el uso pedagógico de las tecnologías digitales (Prestridge, 2007; Sadaf et al., 2012).

\section{DISEÑO DE INVESTIGACIÓN}

El diseño de investigación utilizado fue de tipo mixto, secuencial y explicativo, implementado en dos fases (Creswell, 2009; Teddlie \& Tashakkori, 2009). La primera, fue destinada a caracterizar los usos, creencias pedagógicas y nivel de innovación con tecnologías digitales manifestada por una muestra aleatoria simple de 116 docentes de la carrera de pedagogía en educación básica (primaria) de 21 universidades chilenas (tabla 1). 
Tabla 1. Distribución de Muestra de Participantes Fase 1

\begin{tabular}{|l|c|c|c|}
\hline \multicolumn{1}{|c|}{ Universidad } & Mujeres & Hombres & Total \\
\hline PUCV & 14 & 4 & 18 \\
\hline U. Central & 6 & 4 & 12 \\
\hline U. de Los Lagos & 5 & 6 & 11 \\
\hline UCSC & 4 & 5 & 9 \\
\hline UCSC & 4 & 5 & 9 \\
\hline UCINF & 4 & 4 & 8 \\
\hline U. Miguel de Cervantes & 6 & 0 & 6 \\
\hline U. de Playa Ancha & 2 & 3 & 5 \\
\hline U. Autónoma de Chile & 3 & 2 & 5 \\
\hline U. del Bío-Bío & 2 & 3 & 5 \\
\hline U. de Tarapacá & 2 & 3 & 5 \\
\hline U. de Atacama & 4 & 0 & 4 \\
\hline U. Bolivariana & 1 & 3 & 4 \\
\hline U. Pedro de Valdivia & 3 & 1 & 4 \\
\hline U. San Sebastián & 2 & 2 & 4 \\
\hline U. Fines Terrae & 3 & 1 & 4 \\
\hline U. Católica de Temuco & 1 & 2 & 3 \\
\hline PUC & 2 & 0 & 2 \\
\hline UMCE & 1 & 1 & 2 \\
\hline U. Los Leones & 2 & 0 & 2 \\
\hline U. Santo Tomas & 0 & 2 & 2 \\
\hline U. República & 67 & 47 & 1 \\
\hline Total & & & 116 \\
\hline
\end{tabular}

El instrumento utilizado para esto corresponde a un cuestionario estructurado conformado por tres dimensiones de información: aspectos socio-contextuales de los informantes, uso de tecnologías digitales y creencias e intenciones pedagógicas, con un total de 131 reactivos (Garrido \& Ponce, 2012). Este instrumento tiene una consistencia interna entre los ítemes, con un alfa de Cronbach de 0,948 .

Las respuestas al cuestionario fueron analizadas a partir de una adaptación al modelo bidimensional pedagógico-tecnológico de integración curricular de las tecnologías digitales, propuesto por Lin, Wang \& Lin (2012). De esta manera, los docentes fueron clasificados de acuerdo a la intencionalidad pedagógica con la cual orientan sus clases, ubicándolos en una de cuatro creencias pedagógicas utilizadas como marco de análisis 
(tabla 2). De la misma manera, se realizó una segunda clasificación de acuerdo al modo de incorporación de las tecnologías digitales en sus clases, ubicándolos en uno de ocho niveles de la escala progresiva de uso de TIC utilizada (tabla 3 ).

Tabla 2. Descripción de la Intención Pedagógica.

\begin{tabular}{|l|l|}
\hline \multicolumn{1}{|c|}{ Creencia Pedagógica } & Descripción de la Clase Intencionada \\
\hline Enseñanza Directiva (ED) & $\begin{array}{l}\text { En mi clase los contenidos los abordo articulando mis exposiciones, } \\
\text { algunas lecturas claves y evaluaciones al final de las unidades en las } \\
\text { cuales cada estudiante debe demostrar cuanto ha aprendido. }\end{array}$ \\
\hline $\begin{array}{l}\text { Actividades Cognitivas } \\
\text { de Aprendizaje (ACA) }\end{array}$ & $\begin{array}{l}\text { En mi clase los contenidos los abordo mediante actividades sesión a } \\
\text { sesión a partir de las cuales solicito a los estudiantes elaborar mapas } \\
\text { conceptuales. }\end{array}$ \\
\hline $\begin{array}{l}\text { Aprendizaje Constructivo } \\
\text { (AC) }\end{array}$ & $\begin{array}{l}\text { En mi clase los contenidos los abordo mediante actividades en las } \\
\text { cuales los estudiantes deben resolver situaciones problemáticas o } \\
\text { desarrollar proyectos de investigación. }\end{array}$ \\
\hline $\begin{array}{l}\text { Aprendizaje Social (AS) } \\
\text { (Teddlie \& Tashakkori) }\end{array}$ & $\begin{array}{l}\text { En mi clase los contenidos los abordo mediante actividades en las } \\
\text { cuales los estudiantes deben debatiry profundizar con otros estudiantes } \\
\text { y personas los temas que deben aprender. }\end{array}$ \\
\hline
\end{tabular}

Fuente: Elaboración Propia, a partir de Lin, Wang \& Lin (2012)

Tabla 3. Escala Progresiva de Uso de TIC

\begin{tabular}{|c|l|}
\hline Nivel & \multicolumn{1}{|c|}{ Descriptor } \\
\hline N1 & No Interesa/No sabe Usarlas \\
N2 & Uso de TIC en pequeñas actividades. \\
N3 & Proyecta información en clases, p.e. usando Power Point \\
N4 & Usa frecuentemente herramientas de comunicación/información. \\
N5 & Diseñar materiales multimediales propios. \\
N6 & Elabora materiales multimediales conjuntamente con estudiantes. \\
N7 & Usa/Crea tutoriales para trabajo autónomo de estudiantes. \\
N8 & Uso plataformas virtuales para actividades colaborativas \\
\hline
\end{tabular}

Fuente: Elaboración Propia, a partir de Lin, Wang \& Lin (2012) 
Las clasificaciones resultantes se cruzaron matricialmente, surgiendo agrupaciones pedagógicas-tecnológicas intencionadas por los docentes analizados, las que representan disposiciones estadísticas sobre el uso de tecnologías digitales en practicas de enseñanza. A modo de ejemplo: docentes que intencionan sus diseños de clases desde un enfoque basado en una "enseñanza directiva" (ED), y que incorporan TIC para "proyectar información utilizando power point" (N3).

La segunda fase estuvo destinada a recopilar, analizar y categorizar descripciones detalladas de buenas y malas prácticas implementadas por los docentes-formadores en aula con tecnologías digitales. El método utilizado correspondió a entrevistas semiestructuradas aplicada a una muestra intencionada de tipo teórica (por saturación) de 40 docentes-formadores representantes de cada una de las agrupaciones de intencionalidad pedagógica surgidas de la primera fase de análisis: Enseñanza Directiva (ED), Actividades Cognitivas de Aprendizaje (ACA), Aprendizaje Constructivo (AC) y Aprendizaje Social (AS), tal como se aprecia en la tabla 4.

Tabla 4. Muestreo teórico final para las entrevistas

\begin{tabular}{|c|c|c|c|}
\hline $\begin{array}{c}\text { Enseñanza } \\
\text { Directiva } \\
\text { (ED) }\end{array}$ & $\begin{array}{c}\text { Actividades Cognitivas } \\
\text { de Aprendizaje } \\
\text { (ACA) }\end{array}$ & $\begin{array}{c}\text { Aprendizaje } \\
\text { Constructivo } \\
\text { (AD) }\end{array}$ & $\begin{array}{c}\text { Aprendizaje Social } \\
\text { (AS) }\end{array}$ \\
\hline 12 & 6 & 15 & 7 \\
\hline
\end{tabular}

Las descripciones obtenidas fueron sistematizadas mediante un análisis de categorías cualitativas, las que arrojan luz sobre la manera en que los docentes-formadores de cada agrupación caracterizan usos concretos de tecnologías digitales en el aula. Posteriormente, las categorías obtenidas analizadas fueron contrastadas entre los grupos, en busca de similitudes y diferencias entre las intencionalidades pedagógicas y el uso dado a las tecnologías.

\section{ANÁLISIS Y RESULTADOS}

\subsection{INTENCIONALIDADES PEDAGÓGICAS Y USOS DE TIC EN AULA}

Tal como lo muestra la tabla 5, la mitad de los docentes $(50.4 \%)$ se inclina por diseños de aula bajo una perspectiva de aprendizaje constructivo, es decir, donde los estudiantes deben asumir responsabilidades mayores sobre su propio aprendizaje, utilizando para esto estrategias como la resolución de problemas o el desarrollo de proyectos de investigación. En ellos los docentes-formadores asumen el rol de mediadores y guías de las actividades dentro del aula, propiciando que los estudiantes construyan su conocimiento individual o grupalmente. Si se considera el $19.4 \%$ que señala intencionar su docencia bajo una perspectiva construccionista, se puede concluir que el $69.9 \%$ de los informantes afirma diseñar clases en las cuales el rol protagónico lo tienen los estudiantes. 
Estudios Pedagógicos XLIV, $\mathrm{N}^{\circ}$ 3: 253-269, 2018

INTENCIÓN Y PRÁCTICA CON TIC EN FORMADORES DE PROFESORES: CONGRUENCIAS, COLISIONES Y AUTOEFICACIA

Tabla 5. Distribución de Intencionalidad Pedagógica

\begin{tabular}{|l|c|}
\hline \multicolumn{1}{|c|}{ Creencia Pedagógica } & Distribución de Docentes \\
\hline Aprendizaje Constructivo (AC) & 50.44 \\
\hline Enseñanza Directiva (ES) & 25.66 \\
\hline Aprendizaje Social (AS) & 19.47 \\
\hline Actividades Cognitivas de Aprendizaje (ACA) & 4.42 \\
\hline
\end{tabular}

El 25.6\% de los docentes declaran su preferencia por diseños directivos, en los cuales son ellos quienes asumen el protagonismo en la gestión y desarrollo del ambiente de enseñanza. En otras palabras, los temas se abordan articulando la propia exposición de las materias, recomendando lecturas a sus estudiantes y realizando evaluaciones sumativas, en las cuales los estudiantes deben evidenciar lo aprendido. Si a éstos se suma el $4.4 \%$ que señala diseñar clases bajo una perspectiva cognitiva, en la cual la evidencia de lo aprendido se pone de manifiesto en elaboraciones individuales que permitan mostrar el pensamiento de los estudiantes, entonces se puede afirmar que un tercio de los informantes (29.9\%) intenciona su desempeño en las aulas de clases con diseños donde su propio rol tiende a ser más protagónico.

La intencionalidad pedagógica declarada es interpretada como una creencia sobre la manera en que se concibe el aprendizaje, y sobre el rol que en ello juegan las acciones de docentes y estudiantes. Esto resulta interesante debido a que, a pesar de las diferencias porcentuales constatadas, la preferencia de uso de tecnologías digitales es más bien homogénea. Tal como lo muestra la tabla 6 , los docentes que se agrupan bajo una intencionalidad pedagógica de tipo enseñanza-directiva, utilizan las tecnologías digitales mayoritariamente para la proyección de información utilizando software como power point o prezi (N3), con un $31.03 \%$ y para la comunicación con los estudiantes mediante correo electrónico y en algunos casos plataformas virtuales (N4) con un 31.03\%. Es llamativo señalar que un $10.34 \%$ de los docentes de esta agrupación, también declara utilizar las tecnologías digitales para actividades colaborativas, a través, de entornos virtuales de aprendizaje (N8), o sea el nivel más alto de la escala y que involucra un mayor protagonismo y/o autonomía de los estudiantes.

Tabla 6. Distribución en Escala Progresiva de Intencionalidad Pedagógica de Uso de TIC

\begin{tabular}{|l|c|c|c|c|c|c|c|c|}
\hline \multicolumn{1}{|c|}{ Creencia Pedagógica } & $\mathrm{N} 1$ & $\mathrm{~N} 2$ & $\mathrm{~N} 3$ & $\mathrm{~N} 4$ & $\mathrm{~N} 5$ & $\mathrm{~N} 6$ & $\mathrm{~N} 7$ & $\mathrm{~N} 8$ \\
\hline Enseñanza Directiva (ED) & 3.45 & 10.34 & 31.03 & 31.03 & 6.90 & 6.90 & 0.00 & 10.34 \\
\hline $\begin{array}{l}\text { Actividades Cognitivas de } \\
\text { Aprendizaje (ACA) }\end{array}$ & 0.00 & 0.00 & 40.00 & 40.00 & 20.00 & 0.00 & 0.00 & 0.00 \\
\hline Aprendizaje Constructivo (AC) & 0.00 & 1.75 & 19.30 & 42.11 & 15.79 & 5.26 & 3.51 & 12.28 \\
\hline Aprendizaje Social (AS) & 0.00 & 4.55 & 22.73 & 45.45 & 9.09 & 9.09 & 0.00 & 9.09 \\
\hline
\end{tabular}


El $80 \%$ de los docentes-formadores que declaran intencionar sus clases desde el aprendizaje cognitivo, incorporan las tecnologías digitales de la misma manera que los docentes del tipo enseñanza-directiva (niveles 3 y 4), diferenciándose solo en el uso de TIC para diseñar materiales de tipo multimedial que es utilizado en clases (N5), con un $20.0 \%$.

Los docentes que se declaran en una perspectiva de tipo constructivista, se distribuyen principalmente en 3 niveles de uso de TIC. En un mayor porcentaje (42.1\%) las utilizan para: la comunicación con los estudiantes mediante correo electrónico y en algunos casos mediante plataformas virtuales (N4); la proyección de información utilizando software como power point o prezi (N3), con un 19.3\%; y para diseñar materiales de tipo multimedial que luego usa en sus clases (N5), con el 15.7\%. Solo el 12.2\% señala utilizarlas en actividades de trabajo colaborativo, a través, de entornos virtuales de aprendizaje (N8).

Finalmente, entre los informantes que se ubican en el aprendizaje social (construccionismo), el $45.4 \%$ señala utilizarlas en actividades de enseñanza para el aprendizaje, con énfasis en la comunicación con los estudiantes mediante correo electrónico y en algunos casos plataformas virtuales (N4), mientras que el $22.7 \%$ lo hace para la proyección de información utilizando software como power point o prezi (N3). En éste grupo, solo el $9.1 \%$ las utiliza con énfasis para actividades colaborativas, a través, de entornos virtuales de aprendizaje (N8).

Al comparar los datos de las diferentes agrupaciones analizadas (tabla 7), se puede apreciar que la totalidad de los docentes-formadores, independiente de su intencionalidad pedagógica, indican incorporar tecnologías digitales en sus prácticas en un rango común que se mueve entre los niveles 3 y 5 de la escala de progresión. O sea, sus prácticas declaradas se mueven entre la presentación de información y la preparación de materiales multimediales como recurso de apoyo para las clases.

Tabla 7. Creencias Pedagógicas y Tipos de Uso de TIC de Docentes-Formadores

\begin{tabular}{|l|l|l|l|l|l|l|l|l|}
\hline \multicolumn{1}{|c|}{ Creencia Pedagógica } & N1 & N2 & N3 & N4 & N5 & N6 & N7 & N8 \\
\hline Enseñanza Directiva (ED) & & & & & & & & \\
\hline Actividades Cognitivas de Aprendizaje (ACA) & & & & & & & & \\
\hline Aprendizaje Constructivo (AC) & & & & & & & & \\
\hline Aprendizaje Social (AS) & & & & & & & & \\
\hline
\end{tabular}

Por otro lado, los docentes-formadores del tipo de aprendizaje constructivo, son quienes informan la mayor variedad de usos de tecnologías digitales, señalando realizarlas en los 7 niveles de la escala. Solo entre quienes se agrupan en el tipo enseñanza directiva, se encuentran casos que se ubican en el nivel 1 de la escala, o sea, no las utiliza por desinterés o desconocimiento. Y salvo, en el grupo del tipo aprendizaje constructivo, no se registran docentes que las incorporen para el uso o creación de tutoriales digitales de trabajo destinados al trabajo autónomo de los estudiantes (N7). 


\subsection{DESCRIPCIÓN DE PRÁCTICAS REALIZADAS CON TIC}

El análisis de las entrevistas permite relevar descripciones de prácticas formativas habituales realizadas con el uso o mediación de tecnologías digitales. De acuerdo a las agrupaciones desde las que provienen los informantes, se pueden establecer los siguientes resultados.

\subsubsection{Descripciones grupo Enseñanza Directiva}

Los docentes-formadores pertenecientes a este grupo describen prácticas de aula con tecnologías digitales de acuerdo a dos modalidades. La primera como medio para preparar el contenido de sus clases, mediante la búsqueda de información (p.e. uso de internet): "En Google, todo vía Google, sobre todo información de filosofía, de autores de filosofía actuales, de historia también y de sociología” (DF/G1-I2); la elaboración de presentaciones (p.e. uso de power point o prezi); o "colgando" información en aulas o espacios virtuales a los cuales los estudiantes pueden acceder. La segunda es solicitar tareas a sus estudiantes, en las cuales sea necesario búscar información (p.e. uso de google); preparar y exponer temas tratados en clases (p.e. power point a prezi): "Entonces se expone por grupos y cada grupo tiene que traer su power point” (DF/G1-I5); y ejercitar actividades (uso de aulas virtuales).

Estos profesores describen actividades en las cuales la incorporación de las tecnologías digitales tiene el propósito de motivar, comunicar y desarrollar la habilidad de búsqueda y selección de información. Junto a esto, surgen usos que posibilitan una mayor autonomía en los estudiantes, interactuando individualmente con la información. De esta manera, las prácticas descritas por esta agrupación se mueven principalmente entre los niveles 2,3 y 5 de la escala progresiva de uso de TIC.

\subsubsection{Descripciones grupo Actividades Cognitivas de Aprendizaje}

Los docentes-formadores agrupados aquí, describen prácticas de aula en las cuales las tecnologías digitales son intencionadas principalmente para lo siguiente: Como medio para preparar clases, facilitándo el acceso a información actualizada sobre materias a tratar: "Una de las cosas que más... digamos las hago todas, pero para mí la tecnología me permite buscar información, en términos de materiales, en términos de estudios que se hayan hecho"(DF/G2-P49); para entregar contenidos a los estudiantes mediante presentaciones para complementar y dar soporte a su propio discurso, utilizando para ellos software como power point o prezi:"en la clase ocupo el Power Point por lo general siempre lo ocupo mucho por las imágenes y por imágenes animadas también sobre todo cuando tengo que trabajar los sistemas del cuerpo humano o algunas temáticas con fuerza y movimiento" (DF/G2-P39). Finalmente, para el intercambio de información con los estudiantes fuera del aula, utilizando ambientes como los provistos por redes sociales y/o herramientas de comunicación asincrónica: "Facebook, por lo general ellos se crean su página de Facebook y el curso tiene su página del Facebook y sino hay una alumna que vía Outlook ahora ya no es Hotmail, sí, vía Outlook también nos mandamos harta información.”(DF/G2-P25).

Para estos docentes la incorporación de TIC en las clases tiene el propósito de enseñar a los estudiantes a buscar y seleccionar información, así como propiciar una mayor autonomía en la interacción con los objetos de aprendizajes de las asignaturas: "generalmente intentamos 
que el alumno experimente por sus propios medios ¿cierto?, vamos al laboratorio donde puedan observar, realicen una experiencia sencilla pero sabemos que no todas resultan, no a todos les está resultando como quisieran que ellos obtuvieran en el laboratorio. Por lo tanto, para el cierre de las clases hacemos la simulación mediante el Flash para que ellos vean si el procedimiento que llevaron a cabo coincide o no con lo que realmente esperaba que resultara" (DF/G2-P27). En su conjunto las prácticas descritas por esta agrupación se mueven entre los niveles 2, 3, 4 y 5 de la escala progresiva de uso de TIC.

\subsubsection{Descripciones grupo Aprendizaje Constructivo}

Los docentes-formadores de esta agrupación describen prácticas en las cuales utilizan frecuentemente plataformas virtuales para actividades donde la comunicación directa con los estudiantes adquiere importancia como forma de apoyo al aprendizaje: "está el uso de la plataforma virtual como medio de comunicación para ir colgando información y también para comunicarme con los alumnos" (DF/G3-Y3). El uso de presentaciones cumple el propósito de guiar y ejemplificar el avance de los temas tratados en clases para la mejor comprensión de los estudiantes: "A través del uso de Power Point irles explicando lo que ellas tenían que hacer en la planificación o cuando tenían que mejorar algo en cuanto a los objetivos de alguna actividad, entonces uno va poniendo ejemplos" (DF/G3L12). También describen el diseño y/o uso de simulaciones para potenciar la resolución de problemas o ejemplificar situaciones reales, utilizando para esto herramientas como planillas de cálculo (p.e. Excel), videos y software especializados para que los estudiantes elaboren productos de aprendizaje de manera autónoma.

Para estos informantes, el principal objetivo al incorporar tecnologías digitales, es responder a las exigencias de un nuevo perfil de los estudiantes: usuarios habituales de redes sociales y con capacidades para realizar múltiples tareas. Por esta razón, las descripciones relevan el uso de Facebook como un medio de comunicación e interacción con los estudiantes, paralelo al uso del correo electrónico. Se propicia así el desarrollo de habilidades para buscar, seleccionar, analizar, comunicar y presentar la información, intencionando actividades centradas en el aprender-haciendo; incluso el uso de power point busca ser un medio de discusión o de inicio a trabajos prácticos. Estas prácticas se mueven entre los niveles 2, 3, 5, 7 y 8 de la escala progresiva de uso de TIC.

\subsubsection{Descripciones grupo Aprendizaje Social.}

Las principales prácticas mediada por TIC en este grupo dan cuenta de la preparación de clases, buscando información en internet para actualizar su propio conocimiento sobre las materias que deben tratar y la consiguiente preparación de presentaciones digitales que luego utilizan en clases: "Busco mucha información y preparo mis clases, generalmente utilizando el Power Point porque de esa forma me permite avanzar mucho más rápido, también porque tengo que abarcar una gran cantidad de contenidos y utilizo el power point" (DF/G4-L23). Comunicarse e interactuar con los estudiantes fuera de los horarios de las asignaturas, facilitando una mejor atención formativa, para lo cual utilizan herramientas de comunicación tales como, correo electrónico, Facebook, WhatsApp o mensajes mediante sms: "el principal recurso que utilizamos acá en nuestro quehacer es el correo electrónico, que para nosotros es muy útil, muy útil en relación específicamente para comunicarnos 
con los estudiantes. Tenemos muchos estudiantes nosotros, por lo cual en las clases no son suficientes, por lo cual utilizo bastante cotidianamente el correo electrónico"( DF/ G4-P40). Diseñar situaciones de aprendizaje para movilizar habilidades de pensamiento superior, tales como el análisis, el debate y la elaboración de representaciones de lo aprendido, como sucede con el uso conjunto de dos medios digitales, el cine y los foros de discusión: "da mucho resultado es el cine foro. En el fondo son segmentos de películas o de reportajes que en el fondo están muy bien secuenciados y en base a eso se inicia la discusión"( DF/G4-P54).

En el caso de estos docentes-formadores, las descripciones relevan el uso de diferentes fuentes de información: fotografías, videos, filmes descargados desde internet, el uso de software especializados para áreas disciplinarias como matemáticas, y herramientas de autor. El propósito formativo intencionado con la información de tecnologúas digitales es facilitar el análisis, debate, elaboración y representación de objetos de aprendizaje, ubicándose estas prácticas en los niveles 2, 3, 4, 5, 6 y 8 de la escala progresiva de uso de TIC.

\section{DISCUSIÓN}

Los docentes-formadores estudiados, señalan utilizar tecnologías digitales para un conjunto de actividades que resultan comunes: buscar y seleccionar información para preparar (actualizar) las materias que deben abordar en clases, preparar presentaciones de dicha información para su uso durante la clase o comunicarse con sus estudiantes fuera del horario de clases. Esto implica una base de uso común de tecnologías que no se vincula al tipo intencionalidad pedagógica que poseen los docentes-formadores. Por otro lado, se identifican algunas prácticas con TIC que estan en niveles altos de la escala de progresión utilizada, tales como el diseño de materiales multimediales propios (N5) y el uso de plataformas virtuales para actividades colaborativas (N6), que en algunos casos no resultan congruentes con el tipo de intencionalidad pedagógica expresada.

¿Qué tipo de relación se puede establecer entre las intencionalidades pedagógicas, el uso de TIC descrito y la propia autoeficacia de los docentes-formadores?

Para responder a esta pregunta, es necesario analizar el uso de tecnologías digitales no solo como la consecuencia de una creencia, sino que como una creencia en sí misma, y por tanto, como lo han señalado Loveless (2003) y Prestidge (2012), en coexistencia con otras. De esta forma, los resultados pueden analizarse desde un eje de congruencia-colisión de dos tipos de intencionalidades: las pedagógicas propiamente tales y las referidas al aporte de las TIC como innovación, conformando así un sistema de creencias que influye sobre la autoeficacia.

De esta manera, hay una relación de congruencia o colisión respecto de cuatro elementos a partir de los cuales se despliega la autoeficacia de los docentes-formadores para utilizar las tecnologías digitales en su desempeño: protagonismo del docente, autonomía del estudiante, TIC como medio de colaboración y TIC como medio de reproducción, originando las siguientes relaciones:

- Congruencia entre Protagonismo Docente y TIC Reproductivas. Esta relación corresponde a quienes comparten su creencia en diseños de aulas donde el protagonismo está centrado en quien enseña (docente-formador), e intenciona usos 
de tecnología digital, para la preparación de las clases y la presentación (transmisión) de información ya sea presencialmente o a través de ambientes virtuales. Bajo esta relación se ubican docentes-formadores de todas las agrupaciones de intencionalidad pedagógica, quienes describen usos entre los niveles 2 y 5 , es decir, para actividades acotadas, preparación de clases, presentación de información, comunicación con estudiantes y diseño de materiales digitales multimediales, que en su conjunto soportan o fortalecen su rol protagónico en clases.

- Colisión de Protagonismo Docente-TIC Colaborativas. Corresponde a quienes creen en diseños de aula con un protagonismo centrado en quien enseña (docenteformador), pero que utilizan herramientas o ambientes digitales que requieren de autonomía o colaboración de los estudiantes. Bajo esta relación se ubican docentes-formadores que se identifican con la intencionalidad pedagógica del tipo enseñanza directiva, quienes describen usos entre los niveles 6 y 8 , o sea, incorporan TIC para la elaboración de materiales multimediales conjuntamente con estudiantes y utilizan plataformas virtuales para actividades colaborativas.

- Colisión de Autonomía del Estudiante-TIC Reproductivas. Esta relación corresponde a quienes comparten la creencia en diseños de aulas que otorgan una mayor autonomía al estudiante, pero que intencionan usos con herramientas o ambientes digitales que son reproductivos o de bajo despliegue de habilidades de orden superior. Aquí se ubican quienes se identifican con creencias pedagógicas de aprendizaje constructivista y de aprendizaje social, y que describen usos de TIC para la preparación de clases, proyección de presentaciones en aula, uso de recursos de información y diseño de materiales multimediales para el aula, o sea, en los niveles 2, 3, 4 y 5 de la escala de progresión.

- Congruencia entre Autonomía del Estudiante-TIC Colaborativas. Corresponde a quienes comparten su creencia en diseños de aulas que asignan una mayor autonomía al propio estudiante, y que intencionan usos de tecnología digital que permiten el trabajo individual o colectivo autónomo, en el cual se despliegan habilidades de orden superior, sea dentro o fuera de la clase presencial. Se ubican aquí, los docentes-formadores de las creencias pedagógicas de aprendizaje constructivo y aprendizaje social, quienes describen usos de TIC de los niveles 6, 7 y 8; es decir, para elaborar materiales multimediales con los estudiantes dentro de las clases, usar o crear tutoriales para el trabajo autónomo de los estudiantes y utilizar plataformas virtuales para propiciar actividades colaborativas.

\section{CONCLUSIONES}

Existen evidencias que muestran que las tecnologías digitales tienen una potencialidad para desarrollar aprendizajes y para enriquecer los escenarios formativos (Bartolomé, Cano \& Compaño, 2011; García-Valcárcel \& Tejedor, 2017; Garrido, 2013; Jones, 2012; Marzo, Esteban \& Gargallo, 2006); sin embargo, sus aportes efectivos al mejoramiento de las prácticas de aula aún resultan episódicas (Bates, 2009; Enochsson \& Rizza, 2009; 
Savage \& McGoun, 2012). No obstante, considerando su presencia extendida en el sistema educacional, es necesario preguntarse sobre la manera en que las nuevas generaciones de profesores están siendo formados para este desafío formativo e innovador.

Conscientes del papel que tienen los docentes-formadores de carreras de pedagogía en la mediación de aprendizajes y en el modelamiento de repertorios de actuación profesional para el uso de TIC (Hammond et al., 2009; Schön, 1992; Tondeur et al., 2016; Tondeur et al., 2012; Valtonen et al., 2015), es que esta investigación ha puesto su atención en las intencionalidades y usos que aquellos dan a las tecnologías digitales para sus actividades formativas. El supuesto que subyace es la importancia que tienen las creencias (como pensamiento primario) en la conformación de las intenciones y decisiones que se dan forma a las prácticas formativas, y por tanto, en la autoeficacia que un docente manifiesta para emprender determinadas tareas.

Los resultados obtenidos permiten establecer que la autoeficacia de los docentesformadores se mueve entre la congruencia y la colisión de una determinada creencia de caracter pedagógica-didáctica que actúa como creencia ancla, a partir de la cual se intactúa con otra tipología de creencia de tipo tecnológica que se manifiesta como una creencia fragil (Kagan, 1992). Esto conlleva en que una misma persona desarrolla experiencias formativas en las cuales la incorporación (resignificación) de las TIC resulta congruente con su mirada pedagógica, junto a otras experiencias en las cuales la relación refleja una colisión.

Tal constatación, implica un análisis de la autoeficacia, desde una relación recursiva y compleja, en la cual, es la resultante de un cuerpo de prácticas comunes de uso de las tecnologías digitales, p.e. la preparación y uso de presentaciones digitales en clases, que es transversal al tipo de creencia pedagógica del docente-formador, pero que conviven con otras maneras de utilizarlas, aunque menos frecuentes, y cuyo sentido de uso se va diferenciando de acuerdo al mayor protagonismo que asume el docente o que se otorga al estudiante.

Desde esta óptica, los docentes tienen disposición para implementar o experimentar mayor variedad de uso de tecnologías en sus prácticas de aula, en las cuales los estudiantes asuman un mayor protagonismo, no obstante, la baja frecuencia de esto, incluso entre docentes-formadores con creencias que valoran este protagonismo, permiten inferir bajos niveles de autoeficacia para emprender usos mas innovadores de TIC.

Finalmente como consecuencia de lo anterior, mejorar la autoeficacia de los los docentes-formadores para modelar maneras más innovadoras de usar pedagógicamente las tecnologías digitales en el aula, a partir de las intenciones y creencias pedagógicas, requiere resignificar las estrategias de perfeccionamiento desde una mirada matricial y progresiva a la mejora de sus capacidades. Por un lado, promoviendo cambios en las creencias pedagógicas desde las cuales se conciben la forma de enseñanza para el aprendiaje, por otro lado, ejemplicando variedad de usos formativos y contextualizados a tecnologías digitales disponibles, que ofrezcan un mayor potencial cognitivo.

\section{REFERENCIAS BIBLIOGRÁFICAS}

Aprea, G. (2006). Las representaciones de las TIC en relación con los procesos educativos. En R. Cabello (Ed.), "Yo con la computadora no tengo nada que ver". Un estudio de las relaciones entre los maestros y las tecnologías informáticas en la enseñanza (pp. 89-124). Buenos Aires: Prometeo - Universidad Nacional de General Sarmiento. 
Arancibia, M., Soto, C.P. \& Contreras, P. (2010). Concepciones del profesor sobre el uso educativo de las tecnologías de la información y comunicación (TIC) asociadas a procesos de enseñanzaaprendizaje en el aula escolar. Estudios Pedagógicos, XXXVI(1), 23-51.

Bartolomé, A., Cano, E. \& Compaño, P. (2011). Los Blogs como instrumentos de evaluación de competencias. En T. Pagés, A. Cornet y J. Pardo (Eds.), Buenas prácticas docentes en la universidad. Modelos y experiencias en la Universidad de Barcelona (pp. 77-85). Barcelona: Octaedro/ICE-UB.

Bates, A. (2009). ¿Se comprende realmente lo que es el E-Learning? In A. Gewerc (Ed.), Políticas, prácticas e investigación en tecnología educativa (pp. 109-132). Barcelona: Octaedro/ICE-UB.

Brun, M. (2011). Las tecnologías de la información y las comunicaciones en la formación inicial docente de América Latina. Santiago: CEPAL.

Clark, C.M. \& Peterson, P.L. (1986). Teachers' througth processes. In M. C. Wittock (Ed.), Handbook of research on teaching (3rd. ed., pp. 255-296). New York: Macmillan.

Cortez, K., Fuentes, V., Villablanca, I. \& Guzmán, C. (2013). Creencias docentes de profesores ejemplares y su incidencia en las prácticas pedagógicas. Estudios Pedagógicos, XXXIX(2), 97 113.

Creswell, J. (2009). Research design: Qualitative, quantitative, and mixed methods approaches. Los Angeles-London-New Delhi-Singapore: Sage.

Cuban, 1., Kikpatrick, H. \& Peck, C. (2001). High access and low use of technologies in high school classrooms: explaining and apparent paradox. American Educational Research Journal, 38(4), 813-834.

Den Exter, K., Rowe, S., Boyd, W. \& Lloyd, D. (2012). Using Web 2.0 technologies for collaborative learning in distance education- case study from an Australian University. Future Internet, 4(1), $1-19$.

Duart, J., Gil, M., Pujol, M. \& Castaño, J. (2008). La universitat a la societat xarxa. Barcelona: Ariel.

Enochsson, A. \& Rizza, C. (2009). ICT in Initial Teacher Training: Research Review. doi:https://doi. org/10.1787/220502872611

Ertmer, P., Ottenbreit-Leftwich, A. \& Tondeur, J. (2015). Teachers' beliefs and uses of technology to support 21 st-century teaching and learning. In H. Fives \& M. Gregorie Gill (Eds.), International handbook of research on teaching beliefs (pp. 403-418). New York - Oxon: Routledge.

Flores-Lueg, C. \& Roig Vilab, R. (2016). Percepción de estudiantes de Pedagogía sobre el desarrollo de su competencia digital a lo largo de su proceso formativo. Estudios Pedagógicos, XLII(3), 129-148.

García-Valcárcel, A. \& Tejedor, J. (2017). Percepción de los estudiantes sobre el valor de las TIC en sus estrategias de aprendizaje y su relación con el rendimiento. Educación XX1, 20(2), 137-159.

Gargallo, B., Suárez, J. \& Almerich, G. (2006). The influence of teachers' attitudes in the use of the new technologies. Revista Española de Pedagogía, LXIV(233), 45-66.

Garrido, J. (2013). Videojuegos de Estrategias: Algunos principios para la enseñanza. Revista Electrónica de Investigación Educativa., 15(1), 62-74.

Garrido, J. \& Ponce, C. (2012). Inventory of ICT beliefs in initial teacher training: Toll for identify trends of innovation. Paper presented at the 5th International Conference of Education, Research and Innovation, ICERI, Barcelona.

Hammond, M., Crosson, S., Fragkouli, E., Ingram, J., Johnston-Wilder, P., Johnston-Wilder, S., Kingston, Y., Pope, M. \& Wray, D. (2009). Why do some student teachers make very good use of ICT? An exploratory case study. Technology, Pedagogy and Education, 18(1), 59-73.

Jimoyiannis, A. \& Komis, V. (2007). Examining teachers' beliefs about ICT in education: Implications of a teacher preparation programme. Teacher Development, 11(2), 149-173.

Jones, C. (2012). Networked learnign, stepping beyond the net generation and digital natives. In L. Disckinck-Holmfeld, V. Hodgson, \& D. McConnell (Eds.), Exploring the theory, Pedagogy and practice of networked learning (pp. 27-41). New York-Dordrecht-Heidelber-London: Springer. 
Kagan, D. (1992). Implications of research on teacher belief. Educational Psychologist, 27(1), 65-90.

Kane, R., Sandretto, S. \& Heath, C. (2002). Telling half the story: A critical review of the research into tertiary teachers' beliefs. Review of Educational Research, 72(2), 177-228.

Lee, Y. \& Lee, J. (2014). Enhancing pre-service teachers' self-efficacy beliefs for technology integration through lesson planning practice. Computers \& Education, 73, 121-128.

Liaw, E. (2017). Application of computer-mediated communication on teacher efficacy during school-based field experience. Teaching and Teacher Education, 65, 81-90.

Lim, C. \& Chai, C. (2008). Teachers' pedagogical beliefs and their planning and conduct of computermediated classroom lessons. British Journal of Educational Technology, 39(5), 807-828.

Lim, C. Chai, C. \& Churchill, D. (2010). Leading ICT in education practices: A capacity-building toolkit for teacher education institutions in the Asia-Pacific. Singapore: Microsoft.

Lin, J., Wang, P. \& Lin, I. (2012). Pedagogy* technology: A two-dimensional model for teachers' ICT integration. British Journal of Educational Technology, 43(1), 97-108.

Loveless, A. (2003). The interaction between primary teachers' perceptions of ICT and their pedagogy. Education and Information Technologies, 8(4), 313-326.

Marzo, M., Esteban, L. \& Gargallo, A. (2006). ¿Inciden las nuevas tecnologías en los resultados alcanzados por los alumnos? Un estudio exploratorio. Revista de Educación(340), 695-711.

Newell, D., Pembroke, M. \& Boyd, W. (2012). Crowd sourcing for conservation: Web 2.0 a powerful tool for biologists. Future Internet, 4(2), 551-562.

Prestridge, S. (2007). Engaging with the transforming possibilities of ICT. Australian Educational Computing, 22(2), 3-9.

Prestridge, S. (2012). The beliefs behind the teacher that influences their ICT practices. Computers \& Education, 58(1), 449-458.

Rienties, B., Brouwer, N. \& Lygo-Baker, S. (2013). The effects of online professional development on higher education teachers' beliefs and intentions towards learning facilitation and technology. Teaching and Teacher Education, 29, 122-131.

Sadaf, A., Newby, T. \& Ertmer, P. (2012). Exploring pre-service teachers' beliefs about using Web 2.0 technologies in K-12 classroom. Computers \& Education, 59(3), 937-945.

Sancho, J. (2012). De TIC a TAC, el difícil tránsito de una vocal. Investigación en la Escuela(64), 19-29.

Sang, G., Tondeur, J., Chai, C. \& Dong, Y. (2016). Validation and profile of Chinese pre-service teachers' technological pedagogical content knowledge scale. Asia-Pacific Journal of Teacher Education, 44(1), 49-65.

Savage, J., \& McGoun, C. (2012). Technology, culture and communication. London-New York:Routledge.

Schön, D.A. (1992). La formación de profesionales reflexivos: Hacia un nuevo diseño de la enseñanza y el aprendizaje en las profesiones. ( $1^{\mathrm{a}}$ ed.). Barcelona: Paidós.

Suaréz, J., Almerich, G., Gargallo, B. \& Aliaga, F. (2013). Las competencias del profesorado en TIC: Estructura básica. Educación XX1, 16(1), 39-62.

Teddlie, C. \& Tashakkori, A. (2009). Integrating qualitative and quantitative approaches to research Handbook of Applied Social Research Methods (2nd ed., pp. 283-317). Thousand Oaks: Sage.

Tondeur, J., Pareja Roblin, N., van Braak, J., Voogt, J. \& Prestridge, S. (2016). Preparing beginning teachers for technology integration in education: ready for take-off? Technology, Pedagogy and Education, 26(2), 157-177.

Tondeur, J., van Braak, J., Sang, G., Voogt, J., Fisser, P. \& Ottenbreit-Leftwich, A. (2012). Preparing pre-service teachers to integrate technology in education: A synthesis of qualitative evidence. Computers \& Education, 59(1), 134-144.

Valtonen, T., Kukkonen, J., Kontkanen, S., Sormunen, K., Dillon, P. \& Sointu, E. (2015). The impact of authentic learning experiences with ICT on pre-service teachers' intentions to use ICT for teaching and learning. Computers \& Education, 81, 49-58. 
Valverde, J., Fernández, M.R. \& Revuelta, F. (2013). El bienhestar subjetivo ante las buenas prácticas educativas con TIC: Su influencia en profesorado innovador. Educación XX1, 16(1), 255-280.

van den Beemt, A. \& Diepstraten, I. (2016). Teacher perspectives on ICT: A learning ecology approach. Computers \& Education, 92-93, 161-170.

Zhao, Y. \& Frank, K. (2003). Factors affecting technology uses in schools: an ecological perspective. American Educational Research Journal, 40(4), 807-840. 
